\title{
Elucidating the Mechanism of LBP-8 Shuttling of Lysosomal Lipids into the Nucleus in C. elegans
}

\author{
Matt Tillman ${ }^{1}$, Meng Wang ${ }^{2}$, and Eric Ortlund ${ }^{1}$ \\ ${ }^{1}$ Department of Biochemistry, Emory University School of Medicine, 1510 Clifton Road, \\ Atlanta, GA 30322 \\ ${ }^{2}$ Baylor College of Medicine, Houston, Texas
}

Lipids not only play a vital role as an energy source and structural component in the cell, but also serve as signaling molecules. Many lipids have been identified as ligands for nuclear receptors, such as PPARs, to regulate transcription, however, it isn't understood how insoluble lipids derived from membranes and organelles are transported into the nucleus. Lipid binding proteins (LBPs) were discovered to solubilize lipids and transport them to the nucleus, serving an integral role in lipid-signaling pathways. Lipid binding protein 8, LBP-8, which is highly expressed in the fat storage tissue of Caenorhabditis elegans, was recently discovered to bind to lipids derived from lysosomes and shuttle them into the nucleus to serve as ligands for NHR-49 and NHR-80, homologs of PPARs, to prolong the lifespan of worms. We have determined the first $1.3 \AA$ high-resolution structure of LBP-8, which has allowed us to identify a structurally conserved nuclear localization signal and amino acids necessary for lipid binding. We have mutated these residues in order to elucidate the mechanism of lysosomal lipid signaling to the nucleus in C. elegans. 